

\section{ÍNDICE}

\section{ÁMBITOS PERSONALES PERSONAL ÁMBITOS}

Crisis de Venezuela: Análisis y perspectivas según los titulares de la prensa argentina, española y china

Venezuela's crisis: Analysis and perspectives in the headlines of Argentine, Spain and China press

Hui Feng Liu

Tratamiento informativo de la violencia de género: asesinatos de mujeres. Análisis de la agencia EFE

Informative treatment of gender violence: murders of women. Analysis of the press agency EFE Rosa Rodríguez Cárcela, Agustín López Vivas

MONOGRAFICOS MONOGRAPHS

Presentación Monográfico. El universo transmedia de los medios de comunicación universitarios: acción dentro y fuera del aula en la sociedad postdigital

Antonia Isabel Nogales-Bocio, Ángels Álvarez villa

El papel transformador de la radio universitaria en materias teóricas ajenas a la comunicación

The transformative role of college radio in theoretical subjects outside communication

Miguel Ángel Díaz Monsalvo

La radiodifusión universitaria: acción discursiva radiofónica para la divulgación de la ciencia University Radio Broadcasting: Radiophonic Discursive Action for the Science Popularization Jorge Sadi Durón, Joel Zapata Salazar

El uso corporativo de Instagram en las universidades privadas españolas. Estudio comparativo de treinta y cinco universidades

The corporate use of Instagram in spanish private universities. Comparative analysis of thirty-five private universities 
La radio universitaria como herramienta de inclusión social: OndaCampus en contextos como la cárcel y barrios desfavorecidos

The university radio as a tool for social inclusion: OndaCampus in contexts like the prison and disadvantaged neighborhoods

Leonor Real Adame, Daniel Martín-Pena, Macarena Parejo Cuéllar

Hacer radio universitaria en la era de YouTube: uso de la plataforma de vídeos a demanda en el contexto mexicano

Make college radio in the age YouTube: use of video on demand platform in the Mexican context Marina Vázquez Guerrero

\section{ARTÍCULOS ARTICLES}

O impresso e o digital nos modelos de negócios de jornais locais: uma análise do Sermos Galiza

The press and digital in business models from local media: Analysis of newspaper Sermos Galiza

Giovanni Ramos

Publicidad y cáncer en la prensa escrita (1903-1912)

Advertising and cancer in the written press (1903-1912)

Laura Almudéver-Campo, Ramón Camaño-Puig

Game rules vs. fandom. How Nintendo's Animal Crossing fan-made content negotiates the videogame meanings

Las reglas del juego vs. el fandom. Cómo el contenido hecho por fans de Nintendo Animal Crossing negocia los significados del videojuego

Jose A. Moreno

La comedia de situación y su análisis textual: evolución de los elementos constructivos del formato

The sitcom and its textual analysis: evolution of the constructive elements of the format

Darío Martín Sánchez

Análisis del discurso emocional de Donald Trump en la campaña electoral de $\mathbf{2 0 1 6}$ Analysis of Donald Trump's emotional speech on the 2016 election campaign 
Innovar, comunicar y transformar (en) la Universidad

Innovate, communicate and transform (at) the University

María Sofía Bernat

288-292

Transparencia en los medios: Un requisito imprescindible para medir la rentabilidad social en radio y televisión

Transparency in the media: An essential requirement to measuresocial profitability in radio and television

Amanda Salazar

Los estudios feministas en comunicación: representación de las mujeres en la revolución tecnológica

Feminist studies in communication: representation of women in the technological revolution 


\title{
Análisis del discurso emocional de Donald Trump en la campaña electoral de 2016
}

\author{
Analysis of Donald Trump's emotional speech on the 2016 \\ election campaign
}

\author{
Laura María Caramelo Pérez, Universidad de Extremadura, \\ Plazuela Ibn Marwan s/n. Badajoz - 06071. \\ Icaramel@alumnos.unex.es | https://orcid.org/0000-0002-9237-1604
}

DOI: http://dx.doi.org/10.12795/Ambitos.2020.i47.13

\begin{abstract}
Resumen
Las emociones están presentes en nuestras relaciones personales, sociales y profesionales, repercuten en la manera de interactuar con los demás, en cómo éstos nos perciben y cómo nosotros los percibimos. Por ello, es importante a la hora de comunicar dominar una serie de habilidades sociales y comunicativas para que la comunicación sea efectiva y el mensaje sea percibido de la manera deseada. Sin embargo, las emociones y su correcta expresión dependerán de los objetivos que cada político quiera alcanzar. Por ello, la comunicación emocional irá siempre vinculada al contexto político y social y a las emociones de la ciudadanía con el objetivo de explotarlas en el discurso político a través la comunicación verbal y no verbal.

En primer lugar, abordamos el tema de las emociones y el papel que juegan en el discurso político, en la persuasión de la ciudadanía y en su comportamiento. Debido a la importancia que tiene en cuanto a comunicación emocional, hemos estudiado la comunicación no verbal como principal herramienta de comunicación afectiva. Posteriormente, analizamos la comunicación no verbal de Donald Trump en diferentes declaraciones institucionales, con el fin de demostrar que la comunicación política y emocional varía en función del contexto y de los objetivos del líder político. Por último,
\end{abstract}


y tras un profundo análisis del discurso, tratamos de poner de manifiesto y justificar la influencia de las emociones en la comunicación política y cómo éstas son determinantes en la percepción del mensaje.

\begin{abstract}
Emotions are present in our personal, social and professional relationships, this, affecting the way we interact with others, about how they perceive us and how we perceive them. Therefore, it is important when communicating with others, to master a series of social and communication skills so that communication is effective and the message is perceived in the desired way. However, the emotions and their correct expression will depended on the objectives that each politician wants to achieve. Therefore, emotional communication will always be linked to the political and social context and to the emotions of the citizenry with the aim of exploiting them in the political discourse through verbal and non-verbal communication.

First of all, we address the subject of emotions and the role they play in the political discourse, in the persuasion of the citizens and their behaviour. Due to its importance in terms of emotional communication, we have studied non-verbal communication as the main tool of effective communication. Subsequently, we analyzed Donald Trump's nonverbal communication in different institutional statements, in order to demonstrate that political and emotional communication varies depending the context and the objectives of the political leader. Finally, after a thorough analysis of the discourse, we try to highlight and justify the influence of emotions in political communication and how these are determinant in the perception of the message.
\end{abstract}

Palabras clave: Emociones, comunicación política, comunicación no verbal, medios de comunicación, Donald Trump

Keywords: Emotions, political communication, non-verbal communication, mass media, Donald Trump

\title{
1. INTRODUCCIÓN
}

Tradicionalmente se les ha restado importancia a las emociones, otorgándole mayor relevancia a la parte racional y objetiva del ser humano. Sin embargo, las emociones indican los estados internos de las personas, sus motivaciones, deseos, necesidades y objetivos que le mueven a actuar. Por ello, las emociones tienen un papel importante en la escena política ya que, durante una campaña electoral, las emociones de candidatos y electores suelen estar a flor de piel. Actualmente, podemos ver en las campañas electorales que los candidatos exponen sus propuestas y posiciones y que éstas satisfacen a su auditorio, sin embargo, son las emociones y aspiraciones que el candidato transmite los componentes determinantes de la campaña. 
Podemos ver que esta relación no es actual, ya que han sido numerosos los políticos que han recurrido a la comunicación emocional para llegar al electorado. Ya desde el siglo pasado y, por poner un ejemplo estadounidense, candidatos como Martin Luther King con su discurso 'I have a dream' consiguió conectar con las emociones, los sentimientos y los sueños de futuro de aquellos quienes los escuchaban, transmitiendo un mensaje de esperanza. No obstante, en contraposición a este recurso se encuentra otra emoción, el miedo, al que otros muchos líderes y movimientos han recurrido a lo largo de la historia política. Y es que, según Redoli, sociólogo y politólogo especializado en comunicación política, este tipo de campaña son actualmente muy habituales, como en las últimas elecciones británicas en las que David Cameron empleó este tipo de discurso. Es precisamente este tipo de discurso el empleado por Donald Trump en las pasadas elecciones presidenciales, quien lejos de emplear mensajes esperanzadores, opta por transmitir miedo en los diferentes actos de campaña y es que, el miedo tiene un efecto movilizador sobre el electorado.

No obstante, para que la comunicación emocional sea efectiva, es necesario saber qué emoción explotar con el discurso creado. Por ello, es necesario conocer el contexto sociodemográfico, político y económico para saber qué emoción emplear en cada discurso, vinculándola a determinados temas que impulsen a la ciudadanía a actuar y a decidir ante las urnas. Por ello, teniendo en cuenta los acontecimientos vinculados al aumento de criminalidad en Estados Unidos en los últimos años, Donald Trump emplea el miedo como eje central sobre el que se articula su discurso: la inmigración ilegal, la llegada masiva de refugiados, el narcotráfico, la comunidad musulmana en Estados Unidos y el terrorismo, entre otros.

Por todo ello, la presente investigación pretende llevar a cabo un análisis de las emociones en el discurso político, en el que tomaremos como sujeto a analizar al líder político estadounidense Donald Trump en diferentes intervenciones mediáticas. Estudiamos todos aquellos aspectos comunicativos a tener en cuenta en este tipo de discurso como son las habilidades sociales, la imagen del líder y la importancia de los medios de comunicación, entre otros.

Enmarcamos el presente trabajo en una investigación de corte cualitativo, en el que realizamos un estudio descriptivo del papel de las emociones en comunicación política a través de la revisión bibliográfica y el visionado y análisis de diferentes intervenciones mediáticas. Con ello se pretende comprobar si la tendencia de recurrir a las emociones, en concreto al miedo, en los discursos políticos traspasa la barrera que existe entre el electorado y el candidato. 


\section{MARCO TEÓRICO}

\subsection{Las emociones}

Todas las emociones tienen una finalidad. Existen emociones más o menos placenteras, pero todas son necesarias en nuestra vida para disfrutar de una autoestima saludable, un comportamiento adecuado y unas relaciones interpersonales satisfactorias. Conocer nuestras propias emociones como las de los demás, expresarlas de forma consciente y emplearlas para modificar determinadas situaciones nos permite avanzar en lo individual y como parte de la sociedad. En la última edición del diccionario de la Real Academia de la Lengua Española (RAE), se define emoción como "alteración del ánimo intensa y pasajera, agradable o penosa, que va acompañada de cierta conmoción somática". De esta definición podemos extraer que las emociones conectan a las personas con su entorno y son capaces de sostener, modificar o alterar dicha conexión (Salovey y Mayer, 1989).

Es necesario, además de tratar el concepto de "inteligencia emocional" la cual nos hace tomar conciencia de las emociones propias y de las de los demás, comprenderlas y utilizar esta información para guiar nuestro pensamiento y nuestras acciones.

En este contexto, destaca la comunicación no verbal por ser la principal herramienta de transmisión de emociones y es que, si en muchas ocasiones no somos capaces de comunicar de manera verbal nuestros sentimientos y sensaciones, los gestos, las miradas, las posturas y la voz sí lo hacen.

\subsubsection{Comunicación no verbal}

En el proceso de cualquier acción comunicativa intervienen el componente verbal y no verbal de la comunicación: el componente verbal se encarga de transmitir información a través de las palabras. Sin embargo, el componente no verbal se encarga de transmitir información mediante un proceso en el que intervienen los gestos, la postura y la voz, entre otros. En este sentido, Rulicki (2012) define la comunicación no verbal como "los gestos, las posturas, las miradas, los tonos de voz y otros signos y señales no verbales que constituyen un lenguaje complementario al de las palabras con el que nos comunicamos de forma constante" (p.13).

Los gestos traducen la opinión que nos causa una persona, situación o tema de conversación en modo de aceptación o rechazo. Por ello, al igual que en determinadas ocasiones no decimos abiertamente lo que pensamos, debemos controlar nuestro comportamiento no verbal para no manifestar lo que realmente sentimos. Esto dependerá en muchas ocasiones de la situación profesional de la persona o del contexto en el que nos encontremos, por lo que es necesario aprender a gestionar y controlar el comportamiento no verbal. 
No obstante, se trata de una tarea compleja en la que hay que atender a todos los aspectos y desde todas las perspectivas de la comunicación no verbal, como son la kinesia, paralingüística y proxémica.

\subsubsection{Kinesia: la importancia de la postura y los movimientos corporales}

Kinesia es el campo que estudia nuestro comportamiento corporal, los movimientos realizados a través del rostro, extremidades superiores e inferiores, confiriendo un significado determinado a nuestro mensaje y sirviendo como refuerzo a nuestra comunicación verbal (Rodríguez, 2010). Esta disciplina atiende a los siguientes aspectos de comunicación corporal:

- Postura: Se pueden diferenciar principalmente entre postura abierta y cerrada. El tipo de postura determinará la predisposición, la aceptación o el rechazo hacia el interlocutor o el tema a tratar.

- Orientación: Establece el ángulo que forma nuestro cuerpo respecto al de nuestro interlocutor. La orientación será más directa o menos directa dependiendo del grado de implicación en la interacción.

- Gestos: Son aquellos movimientos, voluntarios o involuntarios, que se hacen con alguna parte del cuerpo ya sea con el rostro o con alguna articulación. Existen cinco categorías de gestos:

- Emblemas: Son gestos producidos de manera intencionada y son conocidos por la mayoría de la gente.

- Ilustrativos o ilustradores: Son aquellos gestos que refuerzan la comunicación verbal o que sustituyen a una palabra.

- Reguladores: Sirven para sincronizar o regular la interacción comunicativa.

- Gestos que expresan estados emotivos o muestras de afecto: Reflejan estados emocionales en función del mensaje.

- Adaptadores: Suelen ser gestos realizados de manera inconsciente. Reflejan aquellas emociones que no queremos expresar.

- Expresión facial: Son todos aquellos gestos realizados con los ojos, las cejas, párpados, labios y boca. En este sentido, Ekman (1975) considera que el rostro ocupa un lugar primordial en la comunicación de los estados emocionales, refleja actitudes interpersonales, proporciona retroalimentaciones no verbales sobre los comentarios de los demás y algunos aseguran que, junto con el habla humana, es la principal fuente de información. 


\subsubsection{Paralingüística: el poder de la voz}

La paralingüística analiza las variaciones no lingüísticas de la comunicación oral. En este sentido, Fonseca (2010) define la paralingüística como "la disciplina auxiliar de la comunicación no verbal que estudia los elementos vocales y su notable influencia en el desarrollo de las habilidades de la voz en la comunicación oral" (p. 50). Asimismo, el autor advierte una serie de funciones de la voz:

- Volumen: la potencia con la que percibimos un sonido. Un volumen muy elevado denota seguridad y uno muy bajo indica timidez.

- Velocidad: se asocia al estado de ánimo, si estamos tristes hablaremos más despacio que si estamos alegres o nerviosos.

- Tono: se puede modular para expresar diferentes estados de ánimo: alegría, tristeza, ira, etc.

- Ritmo: es la agilidad que se lo otorga al discurso, incluyendo pausas y golpes de voz.

- Pausas: facilitan la comprensión del discurso.

- Énfasis: subraya ideas y conceptos.

\subsubsection{Proxémica: la distancia correcta}

La proxémica es la disciplina que se ocupa de analizar la distribución del entorno inmediato que envuelve a los sujetos de una interacción comunicativa, así como la disposición de los elementos dentro de este espacio (Rodríguez, 2010). Además, la proxémica se encarga de analizar la distancia conversacional que mantiene una persona durante la interacción comunicativa y el concepto de territorialidad. Este término, según Knapp (1982), puede definirse como "la conducta cuya característica es un tipo de identificación con un área determinada que indique la propiedad y la defensa de este territorio ante quienes puedan invadirlo" (p.114).

En relación con este tema, surge el concepto de "espacio defendible", entendiéndose como el espacio que cada uno de nosotros necesitamos para desarrollar ciertas actividades y que defendemos en una interacción comunicativa. La distancia de este espacio variará en función de la relación que tengamos con nuestro interlocutor, el lugar o el contexto en el que nos encontremos. En este sentido surge lo que Edward T. Hall denominó como distancia íntima, social, personal y pública, cada una con su fase cercana y lejana (lbíd.): 
- Distancia íntima: va desde el contacto físico hasta los $45 \mathrm{~cm}$. Se distinguen dos fases:

- Fase cercana: a esta distancia se mantiene contacto físico, se ve un gran número de detalles.

- Fase lejana: va desde 15 a $45 \mathrm{~cm}$. A diferencia de la fase anterior, solo pueden entrar en contacto a través de las manos.

- Distancia personal: es la que mantiene una persona entre sí mismo y los demás. Se pueden distinguir dos fases:

- Fase cercana: va desde 45 a $75 \mathrm{~cm}$. A esta distancia aún es posible tocar a la otra persona.

- Fase lejana: oscila entre los 75 y $120 \mathrm{~cm}$. A esta distancia se sitúan las personas cuando tratan un tema personal. La distancia es similar a la longitud de un brazo estirado. No existe contacto físico.

- Distancia social: en esta fase ya no se distinguen los detalles mínimos del rostro de la otra persona y no existe contacto físico ni se espera que pueda haberlo. El tono de voz es el normal y puede escucharse a una distancia de seis metros.

- Fase cercana: va desde los 120 a los $210 \mathrm{~cm}$. A esta distancia se tiene una visión focal. En esta fase se tratan los temas impersonales, como los tratados en una reunión social casual.

- Fase lejana: va desde 210 a $360 \mathrm{~cm}$. Se perciben los detalles de la piel, de los dientes y de la ropa. A esta distancia se eleva el volumen de la voz y es la que se utiliza a la hora de organizar el mobiliario de un despacho: la silla de la persona visitante estará situada a 2,40 o 2,70 metros de la persona que esté sentada detrás de la mesa.

- Distancia pública: en el trayecto de la distancia personal y social a la distancia pública existen algunas variaciones sensoriales significativas.

- Fase cercana: oscila entre los 3,60 y los 7,50 metros. A esta distancia se sube el volumen de la voz, pero no en su totalidad y la visión del cuerpo empieza a perder su volumen llegando a perecer plano.

- Fase lejana: desde 7,50 metros en adelante. Es la distancia que se utiliza para separar a las personas públicas del resto de ciudadanos. Pero no solo en ese caso, sino que es la utilizada para cualquier persona en una intervención pública en general. 


\subsection{Comunicación política}

Aunque la sociología y el derecho son disciplinas que intervienen en el concepto de comunicación política, esta se refiere a toda comunicación que tiene por objeto asuntos políticos. Según Cotteret (1977), "la comunicación política es un intercambio de información entre los gobernantes y los gobernados, a través de canales de transmisión estructurados e informales" (p.1). En esta definición el emisor y el receptor pueden variar entre gobernantes y ciudadanos, sin embargo, no será comunicación política hasta que el intercambio de información tenga consecuencias sobre el sistema político, ya sean directas o indirectas, mediatas o inmediatas (Reyes et al., 2010).

\subsubsection{Influencia de los medios de comunicación en la comunicación política}

Actualmente, los políticos utilizan los medios de comunicación por el innegable poder que tienen a la hora de comunicar. La audiencia asume la información como auténtica por la veracidad que estos otorgan. En este contexto, López (2000) considera que cada vez que encendemos la radio o la televisión, cada vez que abrimos una revista o periódico alguien está intentando educarnos, convencernos de que compremos un producto o persuadirnos de que votemos a un candidato.

Los medios de comunicación son organizaciones con intereses propios, que en muchas ocasiones salen a la luz al informar sobre temas políticos. Estos intereses se transmiten en la elección de una determinada información para convertirla en noticia, en el hecho de no emitir ciertas informaciones como noticias o la manera en cómo se emite una determinada información. Los intereses de los medios de comunicación vienen determinados por la inserción en el mercado y en la política, y por la manera de cumplir sus funciones profesionales, las cuales se corresponden con informar a los ciudadanos para que estos puedan participar en los asuntos públicos, vigilar el poder y servir como foro de debate y difusión de diferentes grupos para transmitir sus demandas e intereses (O’Quínn et al., 2011).

No obstante, los medios de comunicación tienen la función de abastecer a los ciudadanos como consumidores de información y suele dirigirse a una amplia audiencia de masas. Según Rodríguez (2014), la prensa actúa, en este caso, como vigilante de los gobiernos en detrimento del resto de asuntos públicos de interés.

En este contexto, destaca la televisión como medio para difundir el discurso del candidato, por la capacidad que tiene de llegar a su audiencia a través de la vista y el oído, y el espectador puede captar los mínimos detalles de gestualidad del orador que a través de otros medios no sería posible.

Por ello, el discurso político en la actualidad debe ser un discurso ordenado y adaptado a los medios de comunicación y al público al que se transmite. Aunque la retórica clásica 
se basaba en la representación y gritos, la comunicación política actual sigue siendo retórica, pues al igual que la retórica clásica busca persuadir a la audiencia y generar efectos positivos para el orador.

\section{OBJETIVOS Y METODOLOGÍA}

La presente investigación tiene como objetivo principal definir el papel de las emociones en las estrategias de comunicación empleadas en el ejercicio de la comunicación política, como elemento fundamental en la relación emoción e imagen pública.

Asimismo, mediante el presente estudio perseguimos alcanzar una serie de objetivos específicos $(\mathrm{OE})$ que enumeramos a continuación de manera más detallada:

OE1: Comprobar si Donald Trump incluye las emociones en los diferentes discursos de campaña con el fin de persuadir a un mayor número de electores y obtener un mayor número de votantes.

OE2: Demostrar que los medios de comunicación escogidos por Donald Trump para emitir su discurso emocional actúan como un elemento fundamental en la estrategia de persuasión.

OE3: Comprobar si el contexto socioeconómico, sociodemográfico y sociocultural repercute en la elección de la emoción incluida en el discurso.

OE4: Analizar la capacidad de persuasión que posee el miedo y determinar los efectos que tiene sobre los ciudadanos en el contexto de unas elecciones.

Para ello, y para dar respuesta a la importancia y relación existente entre las emociones y el discurso político, planteamos como objeto de estudio de esta investigación el análisis del discurso no verbal de Donald Trump en la campaña electoral de Estados Unidos de 2016. Se trata, por tanto, de una investigación de corte cualitativo ya que, de acuerdo con Monje (2011), se interesa por comprender el significado de los fenómenos y tiene en cuenta las intenciones y motivaciones de los individuos. La muestra de análisis, en este caso es un sujeto único, correspondiéndose con Donald Trump. Este tipo de investigación se denomina también como diseños de investigación de caso único, Mardin (2001) los define como un análisis de la conducta del individuo cuya respuesta es objeto de estudio.

Tal y como afirman Eiroa y Barranquero (2017), "en la selección del método puede resultar de gran ayuda la consulta de investigaciones similares a fin de tomar prestadas técnicas metodológicas que han resultado útiles, así como conocer sus aciertos y errores" (p. 27). Por este motivo, y a través de la revisión bibliográfica, hallamos técnicas empleadas en investigaciones anteriores como la realizada por María Hernández 
Herrate en su tesis doctoral titulada 'La comunicación no verbal en la proyección mediática de la imagen política de José Luis Rodríguez Zapatero durante el curso político 2006-2007', en la que expone que "a la hora de llevar a cabo la parte práctica de la tesis doctoral, se determinó que la técnica de investigación más adecuada para el análisis del comportamiento no verbal del presidente del Gobierno era el análisis de contenido" (Hernández, 2008, p. 934). No obstante, existe una variante de esta técnica metodológica, el análisis crítico del discurso, que emplearemos para la obtención de todos resultantes de la observación y análisis del comportamiento no verbal de Donald Trump en sus apariciones televisivas.

\subsection{Análisis crítico del discurso aplicado a la comunicación mediática de Donald Trump}

En este contexto, y dada la adecuación a la presente investigación, utilizamos como técnica metodológica el análisis crítico de discurso, una variante del análisis de contenido, el cual según Eiroa y Barranquero (2017) se basa en la valoración del texto desde su producción, una dimensión que proporciona información sobre cuáles son los intereses que subyacen tras el mensaje, quiénes tienen acceso a las estructuras del discurso y de qué manera influyen en el mismo, cuáles son sus posibles efectos y cómo estos contribuyen a la difusión ideológica y de valores. Estas cuestiones han sido determinantes en la elección de esta técnica metodológica por la adecuación con la consecución de objetivos de la presente investigación. Resulta, por tanto, fundamental en el análisis de discurso basado en las emociones que han sido analizadas en las intervenciones mediáticas de Donald Trump.

\subsubsection{Universo o corpus}

Para llevar a cabo el análisis crítico de discurso es necesario establecer el universo de estudio. En este caso no hablamos de universo sino de corpus ya que, para llevar a cabo esta técnica, lo apropiado es crear un corpus representativo de los productos audiovisuales o grabaciones registradas y posteriormente someterlos a un conjunto de procedimientos interpretativos y de técnicas de refutación (Piñuel, 2002). En este caso, nos basamos en un corpus audiovisual monolingüe y comparable cuyas características son similares, así como los criterios de selección y lengua y cuya composición está constituida por las intervenciones mediáticas de Donald Trump en el periodo de tiempo seleccionado para el análisis, de junio a noviembre de 2016, que por otro lado está enmarcado en la campaña electoral de ese mismo año en Estados Unidos. Será, por tanto, un corpus creado ad hoc, ya que ha sido diseñado para el estudio de un caso concreto. 


\subsubsection{La muestra}

Para analizar las emociones en el discurso político de Donald Trump, hemos establecido aplicar el análisis al lenguaje no verbal del presidente estadounidense, ya que la parte no verbal del mensaje es el principal vehículo transmisor de las emociones, tanto en el ámbito político como fuera de él. El objeto de estudio será precisamente ese, el análisis del comportamiento no verbal de Donald Trump durante los discursos electorales emitidos y recogidos por diferentes cadenas de televisión americanas. En cuanto a la muestra de la investigación, hemos optado por escoger siete vídeos que se corresponden con el primer discurso mediático sobre política exterior ${ }^{1}$, la primera aparición pública tras el atentado de Orlando $^{2}$, su discurso en la Convención Nacional Republicana ${ }^{3}$, el primer ${ }^{4}$, segundo ${ }^{5}$ y tercer $^{6}$ debate televisivo con su oponente Hillary Clinton y su discurso en el acto de investidura como presidente de Estados Unidos ${ }^{7}$. Decidimos escoger estas muestras de análisis por la relevancia que tienen en cuanto a discurso emocional ya que nos permiten ver la evolución de este dependiendo de la situación en la que se encuentra el país y su ciudadanía, y del momento de la campaña en el que se encuentra el candidato.

Cabe destacar que se trata, por tanto, de un estudio transversal en el que se establece un corte en un momento dado del tiempo mientras que los estudios longitudinales realizan mediciones en varias etapas temporales (Igartua, 2006).

\subsubsection{Unidad de análisis}

En este caso, en el análisis crítico de discurso, las unidades de análisis serán, por un lado, los gestos, movimientos y posturas del presidente Trump (kinesia). Por otro lado, los elementos pertenecientes al discurso oral en cuanto a tono de voz, volumen y ritmo (paralingüística), seguido de los aspectos espaciales de sus intervenciones, así como los objetos móviles y de diseño que aparezcan (proxémica).

\section{RESULTADOS}

Tras la elaboración de un exhaustivo análisis del discurso de Donald Trump en diferentes intervenciones mediáticas a lo largo de la campaña electoral, y tras su victoria en las elecciones, hemos podido comprobar que la mayor parte de su discurso se centra en la comunicación y transmisión de emociones, en concreto el miedo, tanto a través del lenguaje verbal como del lenguaje no verbal con el objetivo de llegar y convencer a una mayor parte del electorado.

En este contexto, es necesario destacar un estudio realizado por Alexei Morozov en el que se demuestra que el miedo se contagia a través de los sentidos. La investigación revela que el miedo se contagia a través del olfato, el oído y la vista, algo muy significativo, desde el punto de vista de nuestra investigación en la que lo visto y lo 
escuchado tienen un papel fundamental. Este contagio se da a través de la actividad de la corteza prefrontal, área asociada con la empatía y cuya vinculación es indiscutible ya que en ambos casos se trata de sentir las emociones del otro. En este sentido, y según Morozov, los resultados sugieren que el miedo se puede transmitir a través del lenguaje del cuerpo, los sonidos y los olores. Por ello, el investigador afirma que el contagio puede producirse por televisión y asegura que "los niños que vieron en la televisión los ataques del 11-S son más propensos a traumatizarse ante las adversidades".

Por ello, nos centramos en evaluar el miedo en el discurso de Donald Trump y su intención de hacer sentir esta emoción en los espectadores y público que asiste a los diferentes actos de campaña a través del discurso verbal, las expresiones faciales, las señales de advertencia y la modulación de la voz.

Tras analizar el primer vídeo, en el que Trump habla por primera vez de sus medidas en cuanto a política exterior, hemos podido ver que el discurso verbal del corte elegido aúna temas como la inmigración ilegal, los refugiados sirios, el terrorismo internacional y el ISIS, temas que, para Trump, parecen permanecer indivisibles y ser todos una amenaza para el país. Por ello, no hace diferenciación entre unos y otros, los trata todos a la vez y menciona palabras como "extremismo", "catástrofe" y "ataques". En cuanto a la modulación de la voz, durante la mayor parte del discurso presenta un volumen y un tono medio, y solo en algunas ocasiones eleva y baja el volumen por lo que no resulta demasiado agresivo. En cuanto a las señales de advertencia, eleva en tres ocasiones el dedo índice mientras mira a cámara y al público. Por último, la expresión facial es la mayor parte del tiempo relajada: las cejas relajadas, los párpados bajados y la boca y mandíbula relajadas, solo en algunas ocasiones frunce el ceño, una expresión de ira. Estos datos quedan reflejados de la siguiente manera:

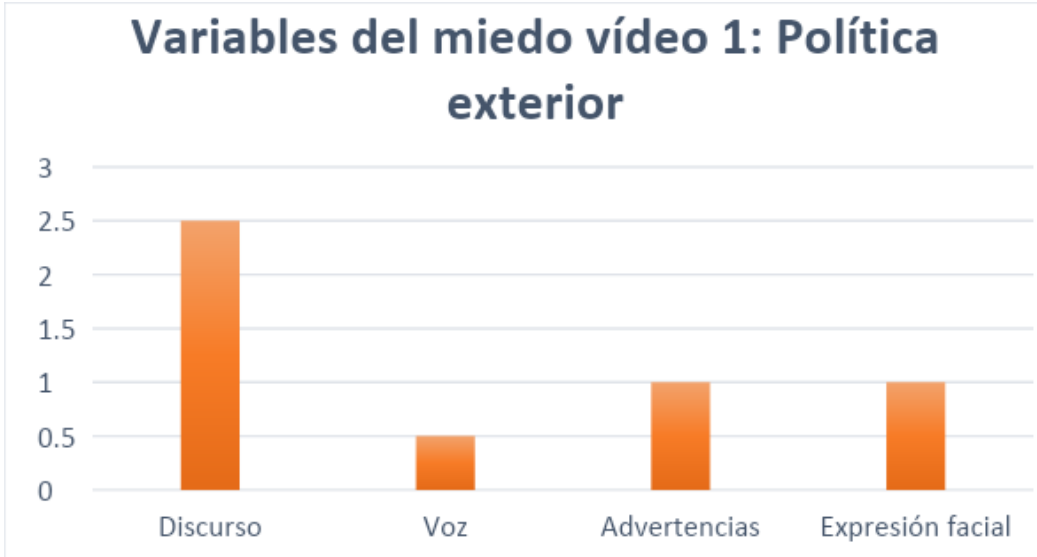

Figura 1. Variables del miedo presentes en el primer vídeo Fuente: Elaboración propia. 
Tras analizar el segundo vídeo, hemos podido comprobar que el discurso que emplea Donald Trump en el corte elegido tras la masacre de Orlando mezcla, al igual que en el anterior, los temas de refugiados sirios, terrorismo internacional e inmigración ilegal, lo que para él es una amenaza para el país mientras emplea palabras como "radicalismo", "devastador", "seguridad", "ataques", "horrible" y "peligrosos". Mientras da su discurso, el volumen de la voz es muy elevado durante la mayor parte, aunque incluye algunas partes en las que el volumen es más moderado sin llegar a ser bajo. En cuanto a las expresiones faciales, la mayor parte del discurso muestra un gesto serio, con el ceño fruncido y la boca abierta expresivamente, mostrando los dientes, un gesto de ira. En cuanto a las señales de advertencia, en este caso señala con el dedo índice en cuatro ocasiones, mirando a cámara y al público. Por ello, el miedo transmitido en este vídeo queda reflejado de la siguiente manera:

\section{Variables del miedo vídeo 2: Atentado de Orlando}

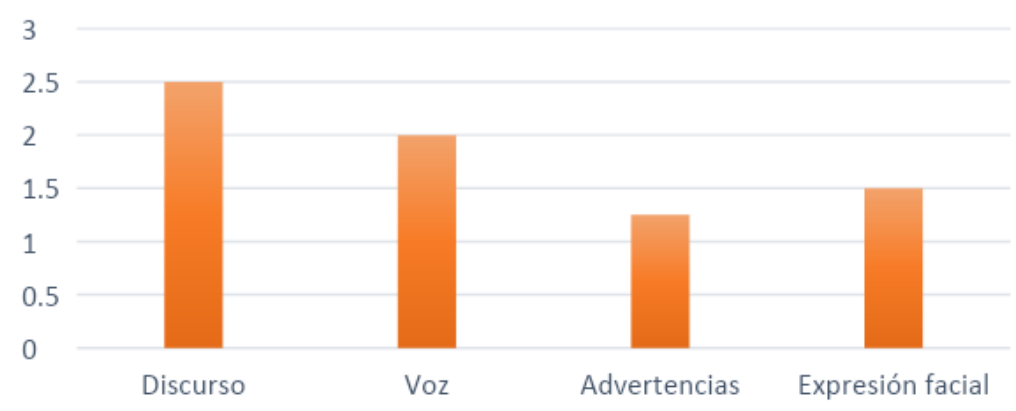

Figura 2. Variables del miedo presentes en el segundo vídeo Fuente: Elaboración propia.

Después de analizar el tercer vídeo, en el que Trump acepta la candidatura oficial a la presidencia por el Partido Republicano en la Convención Nacional, hemos podido comprobar como, de nuevo, en su discurso une en un solo tema el terrorismo internacional, los refugiados sirios y la inmigración ilegal, presentándolos como las amenazas más peligrosas para Estados Unidos y sus ciudadanos mientras emplea palabras como "radical", "violencia" en cuatro ocasiones, "odio", "opresión", "asesinados" en dos ocasiones, "sufrimiento" en tres ocasiones, "protección" en dos ocasiones, "trágico" y "drogas". En cuanto a la modulación de la voz, emplea un volumen muy alto, llegando a gritar en muchas ocasiones, no muestra un volumen moderado ni bajo en ningún momento. En cuanto a las expresiones faciales, durante todo el discurso frunce el ceño y abre la boca de manera expresiva, mostrando una ira y enfado hasta entonces nunca vistos. Por último, respecto a las señales de advertencia, en este caso emplea 27 veces el gesto de señalar mientras mira a cámara y al público. Por todo ello, el miedo transmitido en este discurso queda reflejado de la siguiente manera: 


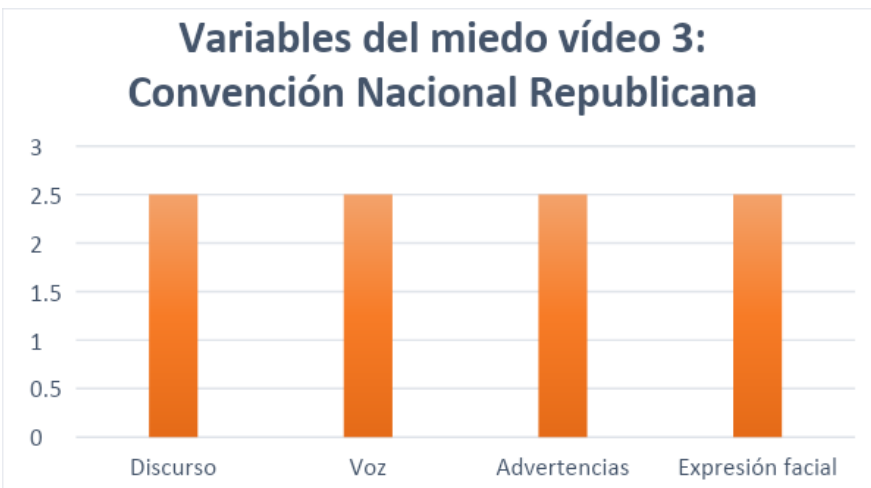

Figura 3. Variables del miedo presentes en el tercer vídeo Fuente: Elaboración propia.

En cuanto al cuarto vídeo, y siendo el primer debate presidencial entre Donald Trump y Hillary Clinton, hemos podido ver como el tono del discurso se suaviza: aunque es cierto que el tema del discurso sigue centrándose en la seguridad del país, en este caso en la inmigración ilegal y en las ciudades más habitadas por hispanos y afroamericanos. Emplea palabras como "peligro", "tiroteos" en cuatro ocasiones, "violencia", "asesinatos" en cinco ocasiones, "criminales" en tres ocasiones y "proteger". En cuanto a la modulación de la voz en ese discurso, comienza con un volumen y tono moderados y a medida que va avanzando su intervención eleva el volumen de la voz, hasta emplear un volumen y tono muy alto. Respecto a las expresiones faciales, muestra en algunos casos el rostro relajado: las cejas relajadas, los párpados bajados y la mandíbula y la boca relajadas. No obstante, en la mayor parte del discurso, frunce el ceño y abre de manera expresiva la boca, una expresión facial, como hemos visto anteriormente de ira y enfado. En cuanto a las muestras de advertencia, señala con el dedo índice en seis ocasiones. Así las cosas, el miedo que transmite Donald Trump en este vídeo queda reflejado de la siguiente manera:

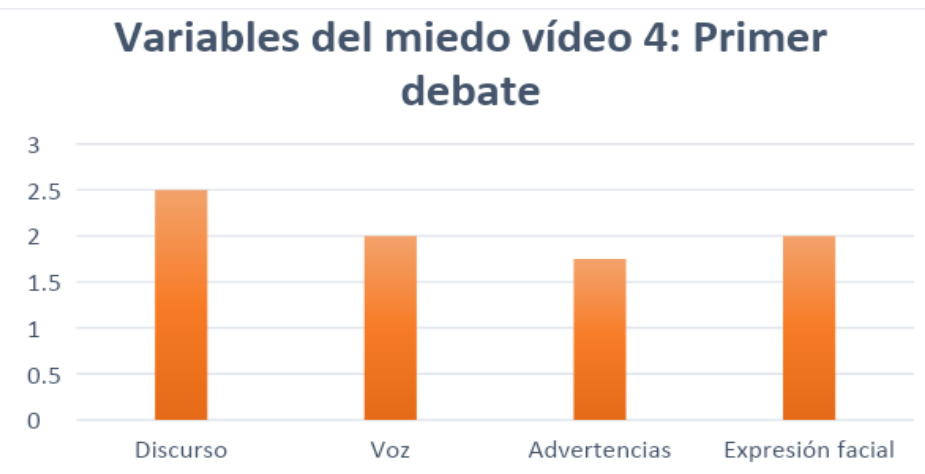

Figura 4. Variables del miedo presentes en el cuarto vídeo Fuente: Elaboración propia. 
Tras analizar el quinto vídeo, correspondiente al segundo debate presidencial entre Donald Trump y Hillary Clinton, hemos podido comprobar que el discurso se suaviza respecto al vídeo anterior. Aunque el tema del discurso oral sigue centrándose en lo que él considera una amenaza para el país, en este caso los refugiados sirios, los inmigrantes ilegales y ahora, las políticas de Hillary Clinton. En este discurso emplea palabras como "criminal" dos veces, "drogas" dos veces y "asesinos". El tono del discurso es más moderado, ya que el volumen durante todo el discurso es medio, llegando a ser bajo en algunos casos. En cuanto a las expresiones faciales, durante la mayor parte del discurso muestra el rostro relajado y solo en algunas ocasiones frunce el ceño y abre la boca de manera expresiva. Por último, respecto a las señales de advertencia, en este caso señala con el dedo índice en ocho ocasiones mientras mira a cámara y a los moderadores del debate. Teniendo todo esto en cuenta, el miedo que transmite Trump en esta intervención queda reflejado de la siguiente manera:

\section{Variables del miedo vídeo 5: Segundo debate}

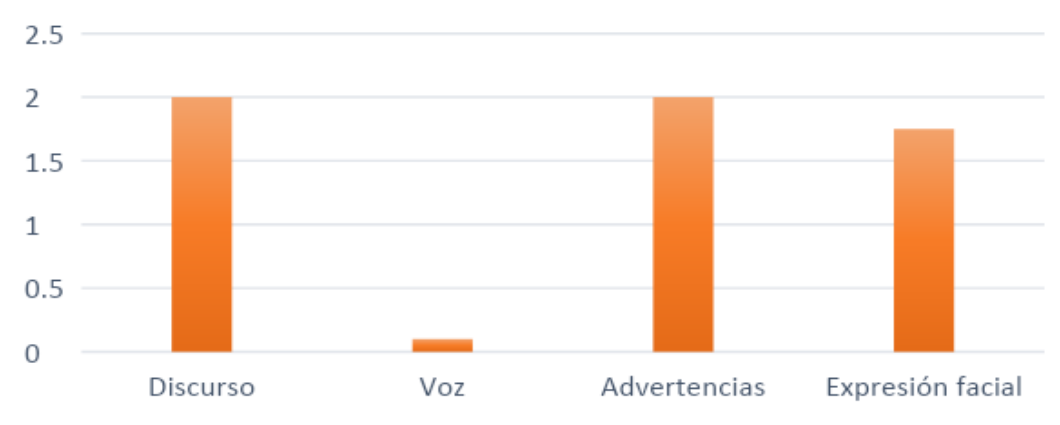

Figura 5. Variables del miedo presentes en el quinto vídeo Fuente: Elaboración propia.

Después de estudiar en profundidad el sexto vídeo, hemos podido comprobar que el discurso tanto verbal como no verbal es semejante al empleado en el segundo debate. Continúa con la tónica de moderar el tono y el volumen del discurso, empleando un volumen medio, sin elevar la intensidad en ningún momento. En cuanto a las expresiones faciales, aparece con un rostro más relajado que en las primeras intervenciones, aunque en algunas ocasiones frunce el ceño, un gesto que indica, como hemos visto anteriormente, enfado. En cuanto al tema del discurso, vuelve a hablar de la inseguridad que hay en el país debido a la inmigración ilegal, a la que ha relacionado con asesinatos, término que menciona en una ocasión, y con las drogas, palabra que menciona hasta en seis ocasiones. En este caso, muestra en dos ocasiones señales de advertencia señalando al público. Por todo ello, el miedo que transmite Trump en el tercer debate presidencial queda representado de la siguiente manera: 


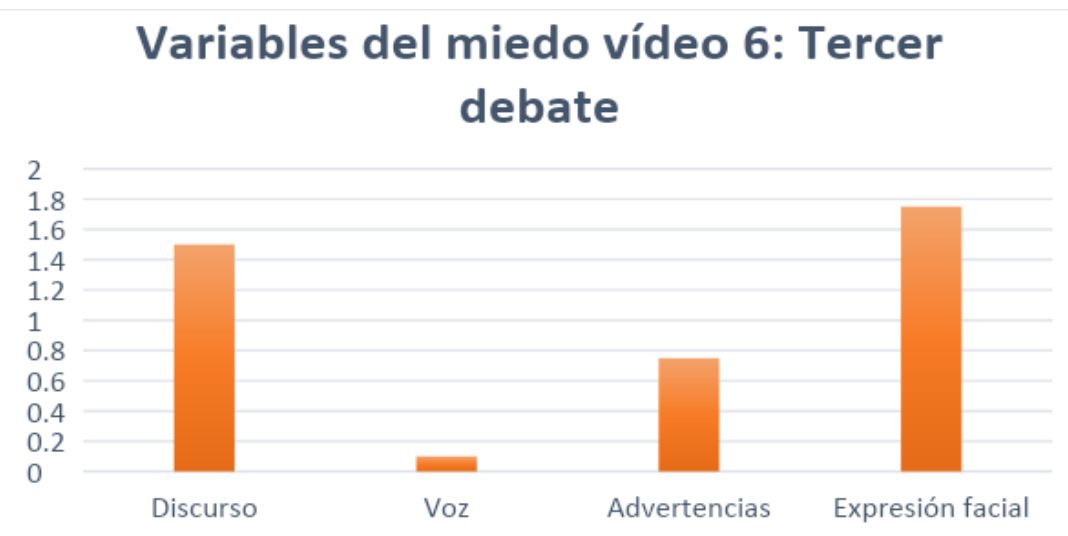

Figura 6. Variables del miedo presentes en el sexto vídeo Fuente: Elaboración propia.

Por último, en cuanto al séptimo vídeo, en el que Donald Trump asiste al acto de investidura, vuelve a elevar el tono del discurso. En este caso, el volumen con el que empieza su discurso es moderado, aunque, en algunas ocasiones, la voz es más elevada que en los debates, pero no llega a ser tan elevada como en las primeras intervenciones. En este caso, el discurso está dividido en varios temas, no como en las ocasiones anteriores, en las que inmigración, terrorismo, refugiados, drogas y criminalidad, eran aunadas en uno solo, para Trump todo significaba lo mismo. Por ello, en este caso, hemos captado diferentes momentos del discurso ya que en un primer momento habla del crimen, las pandillas y las drogas, en un segundo momento habla de la protección de las fronteras de países extranjeros y en un tercer momento habla de terminar con el extremismo islámico. No obstante, en esta ocasión hace referencia al miedo por primera vez y de forma verbal, sin embargo, lo hace para decir que los ciudadanos no deben tener miedo, ya que cuentan con la ayuda de las tropas y la de Dios y cita un fragmento de la Biblia. Este hecho es significativo ya que es a partir de este momento cuando Donald Trump ya es oficialmente presidente de Estados Unidos, por lo que, una vez conseguido su objetivo, insta a los ciudadanos a no tener miedo. En cuanto a las señales de advertencia, señala en un total de nueve veces, sin embargo, en este caso, y a diferencia de las intervenciones anteriores, no advierte a los ciudadanos de los peligros, sino que advierte a quienes él consideraba peligrosos, de que, con él como presidente, esa situación va a terminar. En cuanto a las expresiones faciales, muestra en todo momento el ceño fruncido, un gesto, al igual que en los vídeos anteriores, que muestra ira. Teniendo todo esto en cuenta, el miedo que transmite Trump en este video se refleja de la siguiente manera: 


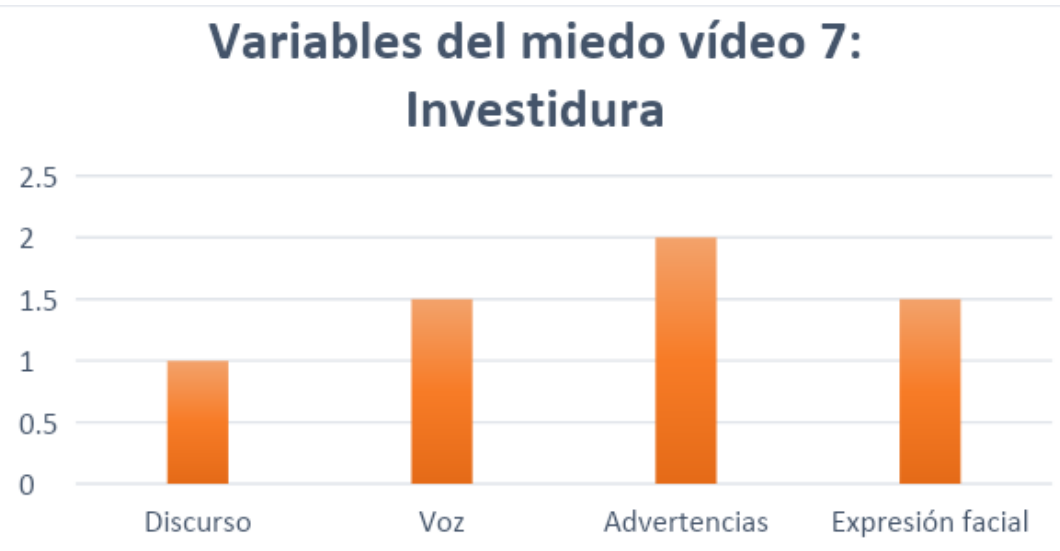

Figura 7. Variables del miedo presentes en el séptimo vídeo Fuente: Elaboración propia.

Tras analizar las variables del miedo que influyen en la transmisión de esta emoción a través del discurso verbal y no verbal de los siete vídeos emitidos por televisión, podemos observar cómo existe una variación de su discurso, dependiendo del momento en el que se encuentra la campaña.

\section{Evolución del miedo en los discursos}

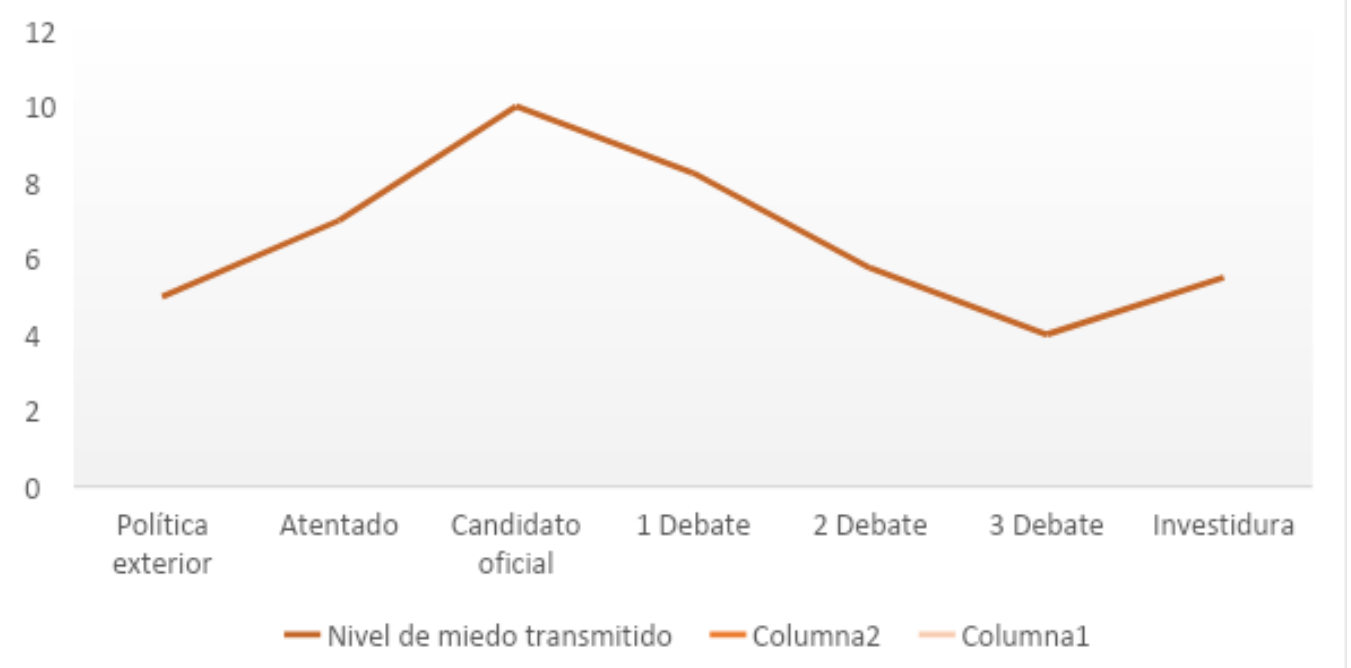

Figura 8. Evolución del miedo en el discurso de Donald Trump de mayo a noviembre de 2016

Fuente: Elaboración propia.

En este sentido, cabe recordar el hecho de que la comunicación no verbal, su análisis y su empleo dependen del contexto, un elemento esencial y que debe ser tenido siempre 
en cuenta. Por ello, es necesario destacar que al igual que hemos tenido en cuenta el contexto para analizar el lenguaje no verbal, Donald Trump tuvo en cuenta el contexto para transmitir distintos niveles de miedo en cada discurso, ya que como podemos observar, después del atentado de Orlando, que tuvo lugar en junio de 2016, Donald Trump aumenta el nivel de miedo tanto en el discurso verbal como en el no verbal. No obstante, el punto más elevado en la transmisión del miedo tiene lugar en la Convención Nacional Republicana que tiene lugar en julio de 2016 en Ohio, un estado que, según las encuestas, sirve de barómetro en cuanto al voto, es decir, el candidato que gana en Ohio gana las elecciones, o al menos eso ha ocurrido en 28 de las últimas 30 elecciones. Del mismo modo ocurre con el segundo momento más elevado en cuanto a la transmisión del miedo, siendo el primer debate presidencial el cual, según diferentes medios de comunicación sería el más visto de la historia con una audiencia de 100 millones de espectadores, por lo que el resto de los debates, aun siendo importantes en cuanto a persuasión, son considerados de menor importancia. Finalmente, y como hemos visto anteriormente, en su primer discurso como presidente apela a la confianza que deben tener en él pues, aunque hace referencia algunos temas que anteriormente había empleado para provocar miedo a la ciudadanía, en este caso los emplea para decir que no deben tener miedo, ya que él ha llegado y acabará con todos esos problemas.

\section{DISCUSIÓN Y CONCLUSIONES}

Después del análisis del discurso llevado a cabo en esta investigación para alcanzar los objetivos previamente planteados al inicio de este artículo y tras la exposición de los resultados, hemos podido establecer una serie de conclusiones que nos llevan a lograr los objetivos planteados al comienzo de este estudio.

Teniendo en cuenta lo anterior, podemos afirmar que Donald Trump basa su discurso en las emociones como estrategia de persuasión hacia el electorado. Conociendo el miedo social que existía en ese momento en Estados Unidos, supo explotar esta emoción en su discurso para convencer a la ciudadanía de que su oponente, Hillary Clinton, no resolvería los problemas que tanto preocupaban a los electores. A su vez, como pudimos ver su discurso estaba enfocado, no solo a tratar el miedo existente entre los electores, sino que, además, creaba nuevos miedos mencionando reiteradamente una peligrosidad que aseguraba que existía. Por ello, podemos decir que a través de su discurso pretendía no solo no mitigar el miedo existente sino fomentarlo para mejorar sus resultados en las urnas el 8 de noviembre de 2016.

Por otro lado, para transmitir su discurso a los electores, emplea fundamentalmente la televisión que funciona como un elemento más en el proceso de persuasión. La televisión es utilizada por el entonces candidato para transmitir el discurso que pronuncia en los actos de campaña, los mítines y los debates electorales ya que tiene 
la capacidad de hacer llegar el mensaje a un gran número de personas y sabe en qué momento explotar en mayor medida su discurso en televisión aprovechando los momentos de máxima audiencia, como pudimos ver en el primer debate presidencial, el cual se esperaba que fuera el más visto de la historia de Estados Unidos y, como pudimos ver, uno de los momentos en los que el índice de miedo es mayor durante la campaña. Por lo tanto, podemos afirmar que la televisión fue un elemento más en la creación del discurso emocional y que, además, fue fundamental en el proceso de persuasión electoral.

La comunicación emocional como estrategia de persuasión en el ámbito político actúa de forma positiva en los resultados electorales. A la vista de los resultados electorales obtenidos, podemos decir que Donald Trump consiguió a través del miedo convencer al electorado, algo que resulta más fácil en un perfil concreto de electores que, por otro lado, coincide con el perfil de votantes de Trump. Los votantes blancos, sin estudios superiores y de entre 45 y 65 años se corresponden con el porcentaje más alto de quienes dieron su voto a Donald Trump, correspondiéndose con el segmento de votantes con más probabilidades de ser persuadidos.

Para el empleo del discurso emocional es trascendental conocer el contexto socioeconómico, sociodemográfico y sociocultural. Es fundamental tener esto en cuenta, porque es importante saber qué emociones son las adecuadas para que el mensaje sea recibido como el candidato espera. En este caso, Donald Trump emplea el miedo para articular su discurso y se basa en él porque sabe que existe una emoción social vinculada a una serie de acontecimientos vividos en Estados Unidos en los últimos años. Concretamente, esto lo pudimos ver en el momento en el que tiene lugar el atentado en la discoteca de Orlando (junio de 2016) ya que Donald Trump muestra un discurso en el que el miedo aumenta considerablemente respecto a las intervenciones anteriores. Asimismo, el miedo que transmite en el discurso aumenta en dos momentos clave de la campaña: la Convención Nacional Republicana, celebrada en Ohio, estado clave para ganar las elecciones, y el primer debate presidencial, el cual se esperaba que fuera el más visto de la historia de los procesos electorales estadounidenses.

Las emociones negativas tienen una mayor capacidad de persuasión que las emociones positivas a corto plazo, sin embargo, son las emociones positivas las que consiguen a medio y largo plazo mejores resultados. Aunque el miedo es una emoción en principio desagradable, es necesaria para alejarnos de los peligros ya que se trata de un mecanismo de adaptación que nos hace reaccionar de manera inmediata. En este sentido, hemos podido comprobar que esto es lo que impulsó a los electores convencidos por el discurso emocional a votar a Donald Trump. No obstante, el impulso a actuar se desvanece con el tiempo, ya que se trata de una reacción a corto plazo que no se prolonga en el tiempo. En este sentido, en enero de2019, la cadena de televisión 
CNN realizaba una encuesta cuyos resultados desvelaban que, si en ese momento se celebraran unas elecciones en Estados Unidos, Donald Trump perdería las elecciones, así como la realizada por el diario 'Público' en agosto de 2019.

Por ello, podemos afirmar que el discurso basado en el miedo fue efectivo en el momento de las elecciones, sin embargo, sus efectos se han desvanecido a lo largo de la legislatura de Trump quien, según los estudios realizados, no ganaría las próximas elecciones.

\section{Notas}

${ }^{1}$ https://edition.cnn.com/videos/spanish/2016/04/27/cnnee-ione-molinares-donald-trump-poltica exterior.cnn/video/playlists/atv-road-to-the-white-house-automated/

${ }^{2}$ https://www.youtube.com/watch?v=BHSUSVxgY70\&t=45s

3 https://www.youtube.com/watch?v=4BTq4b2QIBM

${ }^{4}$ https://www.youtube.com/watch?v=UtD0Gx9lvkl

${ }^{5}$ https://www.youtube.com/watch? $v=-$ UoQaf7SJF0\& $\mathrm{t}=4963 \mathrm{~s}$

${ }^{6}$ https://www.youtube.com/watch?v=M-nMm6tKKE

7 https://www.youtube.com/watch?v=6rUJcKuMebg

${ }^{8}$ https://www.arepadigital.com/demuestran-cientificamente-que-el-miedo-se-contagia/

\section{Referencias}

Blaikie, N.W.H. (1991). A critique of the use of triangulation in social research. Quality and Quantity, (25), 115-136.

Cotteret, J. M. (1977). La comunicación política, gobernantes y gobernados. Argentina: El Ateneo.

Denzin, N.K. (1970). Sociological Methods. A Sourcebook. Chicago: IL: Aldine Publishing Company.

Eiroa, M, y Barranquero A. (2017). Métodos de comunicación científica en la comunicación y sus medios. Madrid: Síntesis.

Ekman, P. (1993). Communication through Nonverbal Behavior: A Sourceof Information About an Interpersonal Relationship. En Tomkins, S. S., y Izard, C. E. (Ed.), Affect Cognition and Presonality (27-36). Nueva York: Springer.

Fonseca, M. (2005), Comunicación oral. Madrid: Pearson.

Hall, E. T. (1963). A System for the Notation of proxemic behavior. American Anthropologist, 65, 207-216. 
Hernández, I. (24 de noviembre de 2016). ¿Por qué se equivocan las encuestas? El Mundo. Recuperado de: https://bit.ly/2rYYrKU última revisión [25-09-2018]

Hernández, M., (2008). La comunicación no verbal en la proyección mediática de la imagen política de José Luis Rodríguez Zapatero durante el curso político 2006-2007 (tesis doctoral). Universidad Complutense de Madrid.

Igartua, J. J. (2006). Métodos cuantitativos de investigación en comunicación. Barcelona: Bosch.

Knapp, M. L. (2008). La comunicación no verbal. El cuerpo y el entorno. Barcelona: Paidós.

Lorenzo, J. (2000). Comunicación no verbal. Periodismo y medios audiovisuales. Madrid: Universitas S.A.

Mardin, A. E. (2001), Métodos de investigación en psicología clínica. México: Pearson Educación.

Monje, C. (2011). Metodología de la investigación cuantitativa y cualitativa. Neiva: Universidad Surcolombiana.

O’Quínn, J. A., Morales, J. M., y Rodríguez, E. (2011). Reflexiones sobre la comunicación política. Espacios Públicos, 14(30), 85-101.

Piñuel, J. L. (2002). Epistemología, metodología y técnicas de análisis de contenido. Estudios de sociolingüística, 3(1), 1-42.

Reyes, M. (2010). Comunicación política y medios en México: el caso de la reforma a la Ley Federal de Radio y Televisión. Revista de Ciencias Sociales, 14 (43), 167-178.

Rodríguez, C. (2014). Sistemas mediáticos comparados. Clasificación comparada del nexo medios y política en Hallin y Mancini. Espacios Públicos, 17 (39), 191-198.

Rodríguez, I. (2010). Lenguaje no verbal. A Coruña: Netbiblo.

Rulicki, S. (2012). Comunicación no verbal. Cómo la inteligencia emocional se expresa a través de los gestos. Barcelona: Granica.

Salovey, P. (1997). What is emotional intelligence? En Salovey, P., y Sluyter, D., (Ed). Emotional Development and Emotional Intelligence: Implications for Educators (3-31). New York: Basic Books.

Wainerman, C., y Sautu, R. (comps.), (1997). La trastienda de la investigación. Buenos Aires: Ediciones Lumiére.

Wolton, D. (1995). La comunicación política: construcción de un modelo. Barcelona: Gedisa. 